\title{
Standard monomials and extremal vector systems
}

\author{
- extended abstract -
}

\author{
TAMÁs MÉszÁros ${ }^{1}$ \\ Freie Universität Berlin \\ tamas.meszaros@fu-berlin.de
}

\author{
LAJOS RÓNYAI ${ }^{2}$ \\ Budapest University of Technology and \\ Economics and MTA SzTAKI \\ lajos@info.ilab.szatki.hu
}

\begin{abstract}
We say that a set system $\mathcal{F} \subseteq 2^{[n]}$ shatters a given set $S \subseteq[n]$ if $2^{S}=$ $\{F \cap S: F \in \mathcal{F}\}$. The Sauer-Shelah lemma states that in general, a set system $\mathcal{F}$ shatters at least $|\mathcal{F}|$ sets. A set system is called shattering-extremal if it shatters exactly $|\mathcal{F}|$ sets. In [9] and [13] an algebraic characterization of shattering-extremal set systems was given, which offered the possibility to generalize the notion of extremality to general finite vector systems. Here we generalize the results obtained for set systems to this more general setting, and as an application, strengthen a result of Li, Zhang and Dong from [8].
\end{abstract}

Keywords: shattering-extremal set systems, standard monomials, Gröbner bases, extremal vector systems

All results of this note are part of the PhD dissertation of Tamás Mészáros. For proofs of the main results see [10].

\section{Preliminaries}

Before getting started with the main definitions, we introduce some notation. Throughout this note $\mathbb{F}$ will stand for a field, and $n$ will be a positive integer. The set $\{1,2, \ldots, n\}$ will be referred to shortly as $[n]$ and its powerset as $2^{[n]}$. Vectors of length $n$ will be denoted by boldface letters, and we denote their coordinates by the same letter indexed by respective numbers, for example $\mathbf{y}=\left(y_{1}, \ldots, y_{n}\right) \in \mathbb{F}^{n}$. For the ring of polynomials in $n$ variables over $\mathbb{F}$ we will use the usual notation $\mathbb{F}\left[x_{1}, \ldots, x_{n}\right]=\mathbb{F}[\mathbf{x}]$. To shorten our notation, for a polynomial $f\left(x_{1}, \ldots, x_{n}\right)$ we will write $f(\mathbf{x})$. If $\mathbf{w} \in \mathbb{N}^{n}$, we write $\mathbf{x}^{\mathbf{w}}$ for the monomial $x_{1}^{w_{1}} \ldots x_{n}^{w_{n}} \in \mathbb{F}[\mathbf{x}]$. For a subset $M \subseteq[n]$, the monomial $x_{M}$ will be $\prod_{i \in M} x_{i}\left(\right.$ and $\left.x_{\emptyset}=1\right)$.

\subsection{Shattering-extremal families}

A set system shatters a given set $S \subseteq[n]$ if $2^{S}=\{F \cap S: F \in \mathcal{F}\}$. The family of subsets of $[n]$ shattered by $\mathcal{F}$ is denoted by $\operatorname{Sh}(\mathcal{F})$. In general we have that $|\operatorname{Sh}(\mathcal{F})| \geq|\mathcal{F}|$ for every set system $\mathcal{F} \subseteq 2^{[n]}$. This statement was proved by several authors independently, and is often referred to as the Sauer-Shelah lemma. For a proof see e.g. [2]. A set systems $\mathcal{F} \subseteq 2^{[n]}$ is shattering-extremal, or $s$-extremal for short, if it shatters exactly $|\mathcal{F}|$ sets, i.e. $|\mathcal{F}|=|\operatorname{Sh}(\mathcal{F})|$. For example, if $\mathcal{F}$ is a down-set (i.e. $H \subseteq F$ and $F \in \mathcal{F}$ imply $H \in \mathcal{F}$ ) then $\mathcal{F}$ is s-extremal, simply because in this case $\operatorname{Sh}(\mathcal{F})=\mathcal{F}$. Many interesting results have been obtained in connection with these combinatorial objects, among others by Bollobás, Leader and Radcliffe in [3], by Bollobás and Radcliffe in [4], by Frankl in [5] and recently Kozma and Moran in [7] provided further interesting examples of s-extremal set systems. For a graph theoretical characterization of s-extremal systems see [11] and [12]. Anstee, Rónyai and Sali in [2] related shattering to standard monomials of vanishing ideals. Based on this, the present authors in [9] and [13] developed algebraic methods for the investigation of s-extremal families, which we recall now briefly.

\footnotetext{
${ }^{1}$ Supported by the DRS POINT Postdoc Fellow program

${ }^{2}$ Research supported in part by NKFIH Grant K115288.
} 


\subsection{Algebraic description of s-extremal families}

Given some set $F \subseteq[n]$, let $v_{F} \in\{0,1\}^{n}$ be its characteristic vector, i.e. the $i$-th coordinate of $v_{F}$ is 1 if $i \in F$ and 0 otherwise. Therefore we can identify a set system $\mathcal{F} \subseteq 2^{[n]}$ with the vector system

$$
\mathcal{V}(\mathcal{F})=\left\{v_{F}: F \in \mathcal{F}\right\} \subseteq\{0,1\}^{n} \subseteq \mathbb{F}^{n}
$$

One can then associate to $\mathcal{F}$ the vanishing ideal $I(\mathcal{V}(\mathcal{F})) \unlhd \mathbb{F}[\mathbf{x}]$, where

$$
I(\mathcal{F})=I(\mathcal{V}(\mathcal{F}))=\left\{f \in \mathbb{F}[\mathbf{x}]: f\left(v_{F}\right)=0 \text { for every } F \in \mathcal{F}\right\} .
$$

Note that we always have $\left\{x_{i}^{2}-x_{i}: i \in[n]\right\} \subseteq I(\mathcal{F})$. The vanishing ideal of a general finite point set $\mathcal{V} \subseteq \mathbb{F}^{n}$ can be defined similarly. For more details about vanishing ideals of finite point sets see e.g. [13].

A total order $\prec$ on the monomials in $\mathbb{F}[\mathbf{x}]$ is a term order, if 1 is the minimal element of $\prec$, and $\prec$ is compatible with multiplication with monomials. One well-known and important term order is the lexicographic (lex) order. Here one has $\mathbf{x}^{\mathbf{w}} \prec_{\operatorname{lex}} \mathbf{x}^{\mathbf{u}}$ if and only if for the smallest index $k$ with $w_{k} \neq u_{k}$ one has $w_{k}<u_{k}$. One can build a lex order based on other orderings of the variables as well, so altogether we have $n$ ! different lex orders on the monomials of $\mathbb{F}[\mathbf{x}]$. Given some term order $\prec$ and a non-zero $f \in \mathbb{F}[\mathbf{x}]$, the leading monomial $\operatorname{Lm}(f)$ of $f$ is the largest monomial (with respect to $\prec$ ) appearing with non-zero coefficient in the canonical form of $f$. For an ideal $I \unlhd \mathbb{F}[\mathbf{x}]$ we denote the set of all leading monomials of polynomials in $I$ by $\operatorname{Lm}(I)$. A monomial is called a standard monomial of $I$ if it is not a leading monomial of any $f \in I . \operatorname{Sm}(I)$ denotes the set of standard monomials of $I$. Standard monomials have some very nice properties; among other things, they form a linear basis of the $\mathbb{F}$-vector space $\mathbb{F}[\mathbf{x}] / I$ and in the case of vanishing ideals of finite set systems they are all square-free monomials. In general for vanishing ideals of finite vectors sets, not merely $0-1$ vectors, their number equals the size of the defining point set and for lex orders they can be computed in linear, $O(n|\mathcal{F}| k)$ time, where $k$ is the number of different coordinates appearing (see [6]).

For an ideal $I \unlhd \mathbb{F}[\mathbf{x}]$ a finite subset $\mathbb{G} \subseteq I$ is called a Gröbner basis of $I$ with respect to $\prec$ if for every $f \in I$ there exists a $g \in \mathbb{G}$ such that $\operatorname{Lm}(g)$ divides $\operatorname{Lm}(f)$. $\mathbb{G}$ is a universal Gröbner basis if it is a Gröbner basis for every term order. Gröbner bases have many nice properties, for details the interested reader may consult e.g. [1].

The first key result in the characterization of s-extremal set systems was the algebraic description of the family of shattered sets, namely that

$$
\operatorname{Sh}(\mathcal{F})=\bigcup_{\text {all term orders }} \operatorname{Sm}(I(\mathcal{F}))=\bigcup_{\text {lex orders }} \operatorname{Sm}(I(\mathcal{F}))
$$

where on the right hand side any square-free monomial $x_{H}$ is identified with the set $H \subseteq[n]$. Since the number of standard monomials of $I(\mathcal{F})$ equals $|\mathcal{F}|$ for every fixed term order, as a corollary we obtain the following proposition.

Proposition $1\left([\mathbf{9},[\mathbf{1 3}]) \mathcal{F} \subseteq 2^{[n]}\right.$ is s-extremal if and only if the standard monomials of $I(\mathcal{F})$ are the same for every term/lex order.

As mentioned earlier, for lex orders $\operatorname{Sm}(I(\mathcal{F}))$ can be computed in linear time, however the number of possible lex orders is $n$ !, and so the above result does not offer directly a method to check the extremality of a set system. However it turns out that we actually need only a significantly smaller collection of lex orders.

Theorem 2 Take $n$ orderings of the variables such that for every index $i$ there is one in which $x_{i}$ is the greatest element, and take the corresponding lex term orders. If $\mathcal{F} \subseteq 2^{[n]}$ is not extremal, then among these we can find two term orders for which the sets of standard monomials of $I(\mathcal{F})$ differ.

Accordingly, by computing the standard monomials for $n$ lex orders we can decide the extremality of a set system in $O\left(n^{2}|\mathcal{F}|\right)$ time.

To continue, for $\mathcal{F} \subseteq 2^{[n]}$ define the downshift by the element $i \in[n]$ as 


$$
D_{i}(\mathcal{F})=\{F \backslash\{i\} \mid F \in \mathcal{F}\} \cup\{F \mid F \in \mathcal{F}, i \in F, F \backslash\{i\} \in \mathcal{F}\} .
$$

It is not hard to see that $\left|D_{i}(\mathcal{F})\right|=|\mathcal{F}|$ and $\operatorname{Sh}\left(D_{i}(\mathcal{F})\right) \subseteq \operatorname{Sh}(\mathcal{F})$, hence $D_{i}$ preserves s-extremality (see e.g. [4]). Downshifts are an important tool in the study of set systems, in particular they can be used to give a possible combinatorial description of the family of standard monomials of the vanishing ideal $I(\mathcal{F})$ for lexicographic term orders. For indices $i_{1}, i_{2}, \ldots, i_{\ell}$ put $D_{i_{1}, i_{2}, \ldots, i_{\ell}}(\mathcal{F}):=D_{i_{1}}\left(D_{i_{2}}\left(\ldots\left(D_{i_{\ell}}(\mathcal{F})\right)\right)\right)$.

Proposition $3([9])$ Let $\mathcal{F} \subseteq 2^{[n]}$ and $\prec$ be a lexicographic term order for which $x_{i_{1}} \succ x_{i_{2}} \succ \cdots \succ x_{i_{n}}$. Then

$$
\operatorname{Sm}(I(\mathcal{F}))=D_{i_{n}, i_{n-1}, \ldots, i_{1}}(\mathcal{F}),
$$

where on the left hand side any square-free monomial $x_{H} \in S m(I(\mathcal{F}))$ is identified with the set $H \subseteq[n]$.

The results about s-extremal families also include a nice connection between s-extremal families and the theory of Gröbner bases. Given a pair of sets $H \subseteq S \subseteq[n]$ we define the polynomial $f_{S, H}(\mathbf{x})=$ $\mathbf{x}_{H} \cdot \prod_{i \in S \backslash H}\left(x_{i}-1\right)$. A useful property of these polynomials is that for a set $F \subseteq[n]$ we have $f_{S, H}\left(v_{F}\right) \neq 0$ if and only if $F \cap S=H$, however much more is true.

Theorem $4\left([\mathbf{9},[\mathbf{1 3}]) \mathcal{F} \subseteq 2^{[n]}\right.$ is s-extremal if and only if there are polynomials of the form $f_{S, H}$, which together with $\left\{x_{i}^{2}-x_{i}: i \in[n]\right\}$ form a universal Gröbner basis of $I(\mathcal{F})$.

We remark that in Theorem 4 it is enough to require a Gröbner basis of the above form for just one term order to have an s-extremal family.

\subsection{Extremal vector systems}

There is a usual way of generalizing the notion of shattering (see e.g. [14]) for collections of vectors from $\{0,1, \ldots, k-1\}^{n}$. Let $\mathcal{V}$ be a class of $[n] \rightarrow\{0,1, \ldots, k-1\}$ functions. We say that $\mathcal{V}$ shatters a set $S \subseteq[n]$ if for every function $\mathbf{g}: S \rightarrow\{0,1, \ldots, k-1\}$ there exists a function $\mathbf{f} \in \mathcal{V}$ such that $\left.\mathbf{f}\right|_{S}=\mathbf{g}$. As previously let $\operatorname{Sh}(\mathcal{V})$ denote the family of shattered sets. In the definition of extremality the Sauer-Shelah lemma played a key role, however in this case we cannot expect a similar inequality to hold. Indeed, as $\operatorname{Sh}(\mathcal{V}) \subseteq 2^{[n]}$, there are at most $2^{n}$ sets shattered, but at the same time the size of $\mathcal{V}$ can be much larger, up to $k^{n}$. This lack of a Sauer-Shelah-like inequality suggests to forget about shattering, and define extremality according to Proposition 1.

Proposition 5 ([10]) If $\mathcal{V} \subseteq\{0,1, \ldots, k-1\}^{n} \subseteq \mathbb{R}^{n}$ is a finite set, then $\operatorname{Sm}(I(\mathcal{V}))$ is the same for every lexicographic term order if and only if $\operatorname{Sm}(I(\mathcal{V}))$ is the same for every term order.

Accordingly we define a finite set of vectors $\mathcal{V} \subseteq\{0,1, \ldots, k-1\}^{n} \subseteq \mathbb{R}^{n}$ to be extremal if $\operatorname{Sm}(I(\mathcal{V}))$ is the same for every lexicographic term order, or equivalently if $\operatorname{Sm}(I(\mathcal{V}))$ is the same for every term order. Proposition 5 was needed to guarantee that the definition of extremality in this general setting is compatible with the special case of set systems. We remark that, although in the above definition $I(\mathcal{V})$ is considered inside $\mathbb{R}[\mathbf{x}]$, our results remain true over an arbitrary field $\mathbb{F}$ and vector systems $\mathcal{V} \subseteq\left\{a_{1}, \ldots, a_{k}\right\}^{n} \subseteq \mathbb{F}^{n}$ (see the universality property of standard monomials in [6]).

For $1 \leq i \leq n$, the $i$-section of $\mathcal{V} \subseteq\{0,1, \ldots, k-1\}^{n}$ for arbitrary elements $\alpha_{1}, \ldots, \alpha_{i-1}, \alpha_{i+1}, \ldots, \alpha_{n} \in$ $\{0,1, \ldots, k-1\}$ is defined as

$$
\mathcal{V}_{i}\left(\alpha_{1}, \ldots, \alpha_{i-1}, \alpha_{i+1}, \ldots, \alpha_{n}\right)=\left\{\alpha \mid\left(\alpha_{1}, \ldots, \alpha_{i-1}, \alpha, \alpha_{i+1}, \ldots, \alpha_{n}\right) \in \mathcal{V}\right\}
$$

Using $i$-sections one can define the downshift at coordinate $i$ in the general case. For any finite point set $\mathcal{V} \subseteq\{0,1, \ldots, k-1\}^{n}, D_{i}(\mathcal{V})$ is the unique point set in $\{0,1, \ldots, k-1\}^{n}$, for which

$$
\left(D_{i}(\mathcal{V})\right)_{i}\left(\alpha_{1}, . ., \alpha_{i-1}, \alpha_{i+1}, . ., \alpha_{n}\right)=\left\{0,1, . .,\left|\mathcal{V}_{i}\left(\alpha_{1}, . ., \alpha_{i-1}, \alpha_{i+1}, . ., \alpha_{n}\right)\right|-1\right\}
$$

whenever $\mathcal{V}_{i}\left(\alpha_{1}, \ldots, \alpha_{i-1}, \alpha_{i+1}, \ldots, \alpha_{n}\right)$ is non-empty, and empty otherwise. For indices $i_{1}, i_{2}, \ldots, i_{\ell}$ let as before $D_{i_{1}, i_{2}, \ldots, i_{\ell}}(\mathcal{V}):=D_{i_{1}}\left(D_{i_{2}}\left(\ldots\left(D_{i_{\ell}}(\mathcal{V})\right)\right)\right.$. Now using these definitions Proposition 3 generalizes naturally to this setting as well. 
Proposition 6 ([9]) Let $\mathcal{V} \subseteq\{0,1, \ldots, k-1\}^{n} \subseteq \mathbb{R}^{n}$ be a finite point set and $\prec$ the lexicographic term order order for which $x_{i_{1}} \succ x_{i_{2}} \succ \cdots \succ x_{i_{n}}$. Then

$$
\operatorname{Sm}(I(\mathcal{V}))=D_{i_{n}, i_{n-1}, \ldots, i_{1}}(\mathcal{V}) .
$$

Note that according to Proposition 6 we could have defined extremal vector systems fully combinatorially as demonstrated by the following corollary.

Corollary 7 A finite point set $\mathcal{V} \subseteq\{0,1, \ldots, k-1\}^{n}$ is extremal if and only if $D_{\pi(n), \pi(n-1), \ldots, \pi(1)}(\mathcal{V})$ is the same for every permutation $\pi$ of $[n]$.

In 9], beside Proposition 6, several other results concerning this general setting were proved, however the general versions of the two main results about set systems, Theorem 2 and Theorem 4 , were missing.

\section{Main results}

A polynomial $f(\mathbf{x}) \in \mathbb{F}[\mathbf{x}]$ is called degree dominated with dominating term $\mathbf{x}^{\mathbf{w}}$ if it is of the form $f(\mathbf{x})=\mathbf{x}^{\mathbf{w}}+\sum_{i=1}^{\ell} \alpha_{i} \mathbf{x}^{\mathbf{v}}$, where $\mathbf{x}^{\mathbf{v}_{i}} \mid \mathbf{x}^{\mathbf{w}}$ for every $i$. By basic properties of term orders we have that the dominating term of such a polynomial is also its leading term for every term order. As an example of a degree dominated polynomial one can consider any polynomial of the form $f_{S, H}$ or for $i=1, \ldots, n$ the polynomial $x_{i}^{2}-x_{i}$, all of them appearing in Theorem 4 .

Theorem 8 ([10]) A finite set of vectors $\mathcal{V} \subseteq\{0,1, \ldots, k-1\}^{n} \subseteq \mathbb{R}^{n}$ is extremal if and only if there is a finite family $\mathcal{G} \subseteq \mathbb{R}[\mathbf{x}]$ of degree dominated polynomials that form a universal Gröbner basis of $I(\mathcal{V})$.

We remark that similarly as in the case of Theorem 4 in Theorem 8 it is also enough to require that $I(\mathcal{V})$ has a suitable Gröbner basis for some term order. Similarly, Theorem 2 also generalizes to this vector setting.

Theorem 9 ([10]) Take $n$ orderings of the variables such that for every index $i$ there is one in which $x_{i}$ is the greatest element, and take the corresponding lex orders. If $\mathcal{V} \subseteq\{0,1, \ldots, k-1\}^{n} \subseteq \mathbb{R}^{n}$ is not extremal, then among these we can find two term orders for which the sets of standard monomials of $I(\mathcal{V})$ differ.

Theorem 9 has several interesting consequences. First of all, it means that in the definition of extremality it would have been enough to require that the family of standard monomials is the same for a particular family of lex orders of size $n$. Next, Theorem 9 just like Theorem 2 for set systems, also results an efficient, $O\left(n^{2}|\mathcal{V}| k\right)$ time algorithm for deciding whether a finite set of vectors $\mathcal{V} \subseteq\{0,1, \ldots, k-1\}^{n} \subseteq \mathbb{R}^{n}$ is extremal or not. Finally, Theorem 9, when considered over an arbitrary field $\mathbb{F}$ and vector systems $\mathcal{V} \subseteq\left\{a_{1}, \ldots, a_{k}\right\}^{n} \subseteq \mathbb{F}^{n}$, allows a strengthening of a result by Li, Zhang and Dong from 8, where they investigated the standard monomials of zero dimensional polynomial ideals.

An ideal $I \triangleleft \mathbb{F}[\mathbf{x}]$ is called zero dimensional if the factor space $\mathbb{F}[\mathbf{x}] / I$ is a finite dimensional $\mathbb{F}$-vector space. It is easy to see that vanishing ideals of finite point sets are special types of zero dimensional ideals.

A term order $\prec$ is called an elimination order with respect to the variable $x_{i}$ if $x_{i}$ is larger than any monomial from $\mathbb{F}\left[x_{1}, \ldots, x_{i-1}, x_{i+1}, \ldots, x_{n}\right]$. As an example one can consider any lex order where $x_{i}$ is the largest variable.

For $1 \leq i \leq n$ let $\prec_{i}$ be an elimination order with respect to $x_{i}$. Part $(2) \Leftrightarrow(3)$ of Theorem 4 in 8 states that if $\mathbb{F}$ has characteristic zero, then the standard monomials of any zero dimensional ideal $I \triangleleft \mathbb{F}[\mathbf{x}]$ are the same for every term order if and only if they are the same for $\prec_{1}, \ldots, \prec_{n}$. We claim that (the general form of) Theorem 9 together with the universality property of standard monomials (see 6]) prove the same result for arbitrary fields. For this we remark, that the proof of Theorem 9 uses only the elimination property of lex orders and the fact that the number of standard monomials of the ideal 
considered is the same for every term order. Accordingly, the result remains true if we substitute the lex orders by arbitrary elimination term orders with respect to the variables and the vanishing ideal $I(\mathcal{V})$ by a zero dimensional ideal $I$. For the second part here note that as the standard monomials form a linear basis of the $\mathbb{F}$-vector space $\mathbb{F}[\mathbf{x}] / I$, their number is the same, namely the dimension of this space, for every term order. With these observations in mind one gets the following form of Theorem 9 , which generalizes part $(2) \Leftrightarrow(3)$ of Theorem 4 from [8] to arbitrary fields instead of fields of characteristic zero.

Theorem 10 ([10]) Let $\mathbb{F}$ be an arbitrary field and for $1 \leq i \leq n$ let $\prec_{i}$ be an elimination order with respect to $x_{i}$. Then the standard monomials of any zero dimensional ideal $I \triangleleft \mathbb{F}[\mathbf{x}]$ are the same for every term order if and only if they are the same for $\prec_{1}, \ldots, \prec_{n}$.

\section{References}

[1] W.W. Adams, P. Loustaunau, An Introduction to Gröbner bases, Graduate Studies in Mathematics, Vol. 3, American Mathematical Society (1994)

[2] R.P. Anstee, L. Rónyai, A. Sali, Shattering News, Graphs and Combinatorics 18:59-73 (2002)

[3] B. Bollobás, I. Leader, A.J. Radcliffe, Reverse Kleitman Inequalities, Proceedings of the London Mathematical Society s3-58:153-168 (1989)

[4] B. Bollobás, A.J. Radcliffe, Defect Sauer Results, Journal of Combinatorial Theory Series A 72:189-208 (1995)

[5] P. FrankL, Extremal set systems, Handbook of Combinatorics Vol. 2, MIT Press, Cambridge (1996)

[6] B. Felszeghy, B. RÁth, L. Rónyai, The lex game and some applications, Journal of Symbolic Computation 41:663.681 (2006)

[7] L. Kozma, S. Moran, Shattering, graph orientations and connectivity, The Electronic Journal of Combintaorics 20(3):P44 (2013)

[8] Z. Li, S. Zhang, T. Dong, Finite sets of affine points with unique associated monomial order quotient bases, Journal of Algebra and its Applications 11(2):1250025 (2012)

[9] T. MÉszÁros, S-extremal set systems and Gröbner bases, Diploma Thesis, Budapest University of Technology and Economics (2010), http://userpage.fu-berlin.de/meszarost/thesiswork.pdf

[10] T. MÉszÁros, Algebraic Phenomena in Combinatorics: Shattering-Extremal Families and the Combinatorial Nullstellensatz, PhD Thesis, Central European University, Budapest (2015), www. etd.ceu.hu/2015/meszaros_tamas.pdf

[11] T. MÉszÁros, L. Rónyai, Shattering-extremal set systems of small VC-dimension, ISRN Combinatorics 2013:126214 (2013)

[12] T. MÉszÁros, L. Rónyai, Shattering-extremal set systems of VC dimension at most 2, The Electronic Journal of Combintorics 21(4):P4.30 (2014)

[13] L. Rónyai, T. MÉszÁros, Some combinatorial application of Gröbner bases, In: F. Winkler (ed.), Algebraic Informatics, CAI 2011, Lecture Notes in Computer Science 6742:65-83, Springer (2011)

[14] A. Shinohara, Complexity of computing Vapnik-Chervonekis dimension and some generalized dimensions, Theoretical Computer Science, 137:129-144 (1995) 